\title{
Missing Elements in Surgical Pathology Reports: Breast, Colon and Stomach Cancers
}

\author{
Maryam Kadivar ${ }^{1}$, Parnian Kheirkhah Rahimabad ${ }^{2 *}$
}

\begin{abstract}
Cancer pathology reports play an important role in choice of patient care. They provide crucial information concerning diagnosis, therapy options, and prognosis. Professional pathology institutions, such as the College of American Pathologists (CAP), have developed checklists to ensure the presence of all the required elements in reports. In this study, 438 surgical pathology reports of patients with breast (148), colon (147), and stomach cancer (143) were evaluated with respect to the presence of mandated elements according to CAP checklists. The most common missing element in all the three types of cancer was 'staging' (73.6,53.1, and 56.6\% in breast, colon, and stomach cancer reports missed 'staging', respectively). The second most missing element was 'tumor site' in breast $(64.2 \%)$ and stomach cancer $(30.1 \%)$, and 'procedure' in colon cancer $(29.3 \%)$. 'Perineural invasion' was the third most missing element in the three types of cancer $(25.7,17.0$, and $22.4 \%$ in breast, colon, and stomach cancer, respectively). Only $11.4 \%$ of reports included all key elements required by CAP. The use of checklists was associated with higher rate of completeness. This study demonstrates that the key elements requiring the information on the requisition forms from the clinicians are commonly missed, leading to ambiguity.
\end{abstract}

Keywords: Pathology report - breast cancer - colon cancer - stomach cancer

Asian Pac J Cancer Prev, 17 (3), 1469-1472

\section{Introduction}

The surgical pathology reports remain the most important means of communication between the clinician and pathologist, transferring crucial information concerning diagnosis, treatment, and prognosis to the clinician (Kempson, 1992; Nakhleh, 2006). They also contain important information useful to the patients, nurses, researchers, and epidemiologists (Goldsmith et al., 2008). The quality of a pathology reports is defined by its accuracy, completeness, timeliness, and usability (Nakhleh, 2006; Srigley et al., 2009). However, completeness of pathology reports is considered as a reflection of its overall quality (Srigley et al., 2009).

Due to the fast growing amount of required information in reports, pathologists could easily miss essential elements (Baskovich and Allan, 2011). In creating solution to this problem, checklists for pathology reporting were established by professional institutions (Idowu et al., 2010; Williams et al., 2015). Checklists are pre-defined templates, containing the necessary minimum set of elements for reporting a specific specimen (Idowu et al., 2010; Williams et al., 2015). The introduction of synoptic protocols for reporting, improved the consistency and completeness of the reports (Baskovich and Allan, 2011; Messenger et al., 2011). Previous studies investigated the adequacy of pathology reports and recommended the routine use of check-list style reporting rather than the narrative form (Kempson, 1992; Zarbo, 1992; Gephardt and Baker, 1996; Idowu et al., 2010).

The College of American Pathologists (CAP) has developed cancer reporting checklists being regularly updated based on current evidence (Baskovich and Allan, 2011; Messenger et al., 2011). A surgical pathology inconsistency with CAP checklists was considered incomplete (Idowu et al., 2010). This study was conducted to evaluate the completeness of surgical pathology reports of breast, colon, and stomach cancer according to CAP checklists and to determine the factors affecting it. The most common missing elements in these reports were also demonstrated.

\section{Materials and Methods}

This cross-sectional study was carried out in five hospitals in Tehran, Iran, using convenience sampling. Two of these five hospitals used a synoptic checklist-style reporting system. Three of the five hospitals used narrative form of reporting. A total number of 438 medical records of patients with breast (148), colon (147), and stomach cancer (143) were collected. All the reports were evaluated for the presence of mandated elements for reporting cancer 
Maryam Kadivar and Parnian Kheirkhah Rahimabad

from CAP checklists. The type and percentage of missing elements of each report were recorded.

Data was analyzed using Statistical Package for Social Science (SPSS) software, version 16. Data was presented as percentage values. Completeness of reports was calculated by dividing the number of mentioned elements by the total number of mandatory elements. Mann-Whitney U and Kruskal-Wallis tests were used to compare completeness of reports across subgroups. A P value $\leq 0.05$ was considered significant. The study was approved by the ethics committee of Iran University of Medical Sciences.

\section{Results}

A total number of 438 pathology reports of breast (148, $33.8 \%)$, colon $(147,33.6 \%)$, and stomach cancer (143, $32.6 \%$ ) were gathered from five hospitals in Tehran, Iran.

Table 1 shows the frequency and type of missing elements in reporting by the cancer types. 'Staging' was the most missing element in of the three cancer types (73.6, 53.1, and 56.6\% of breast, colon, and stomach cancer reports missed 'staging', respectively). The second most missing element was 'tumor site' in breast (64.2\%) and stomach cancer $(30.1 \%)$, and 'procedure' in colon cancer $(29.3 \%)$. 'Perineural invasion' was the third most missing element the three cancer types $(25.7,17.0$, and $22.4 \%$ in breast, colon, and stomach cancer, respectively). In total, 'staging' was the most common missing element $(61.2 \%)$, followed by 'tumor site' $(36.1 \%)$, and 'perineural invasion' $(21.7 \%)$.

Regardless of the reporting format (Figure 1), over $35 \%$ of breast cancer reports had 3 or more missing elements. 52 and $41 \%$ of colon and stomach cancer reports missed one element, respectively. Only $11.4 \%$ of

\begin{tabular}{|c|c|c|c|c|}
\hline & $\begin{array}{l}\text { Breast } \\
\text { Cancer }\end{array}$ & $\begin{array}{l}\text { Colon } \\
\text { Cancer }\end{array}$ & $\begin{array}{c}\text { Stomach } \\
\text { Cancer }\end{array}$ & Overall \\
\hline $\begin{array}{l}\text { Missing } \\
\text { element }\end{array}$ & $(\mathrm{N}=148)$ & $(\mathrm{N}=147)$ & $(\mathrm{N}=143)$ & $(\mathrm{N}=438)$ \\
\hline Procedure & $12.80 \%$ & $29.30 \%$ & $0.70 \%$ & $14.40 \%$ \\
\hline Tumor site & $64.20 \%$ & $13.60 \%$ & $30.10 \%$ & $36.10 \%$ \\
\hline Tumor size & $8.10 \%$ & $2.70 \%$ & $3.50 \%$ & $4.80 \%$ \\
\hline $\begin{array}{l}\text { Histologic } \\
\text { type }\end{array}$ & $2.00 \%$ & $0 \%$ & $2.10 \%$ & $1.40 \%$ \\
\hline $\begin{array}{l}\text { Histologic } \\
\text { grade }\end{array}$ & $4.70 \%$ & $4.80 \%$ & $3.50 \%$ & $4.30 \%$ \\
\hline Margins & $6.10 \%$ & $2.70 \%$ & $1.40 \%$ & $3.40 \%$ \\
\hline $\begin{array}{l}\text { Lymph- } \\
\text { vascular } \\
\text { invasion }\end{array}$ & $17.60 \%$ & $8.20 \%$ & $4.20 \%$ & $10.00 \%$ \\
\hline $\begin{array}{l}\text { Perineural } \\
\text { invasion }\end{array}$ & $25.70 \%$ & $17.00 \%$ & $22.40 \%$ & $21.70 \%$ \\
\hline Lymph nodes & $10.10 \%$ & $2.70 \%$ & $4.20 \%$ & $5.70 \%$ \\
\hline Staging & $73.60 \%$ & $53.10 \%$ & $56.60 \%$ & $61.20 \%$ \\
\hline Laterality & $4.70 \%$ & - & - & $4.70 \%$ \\
\hline
\end{tabular}

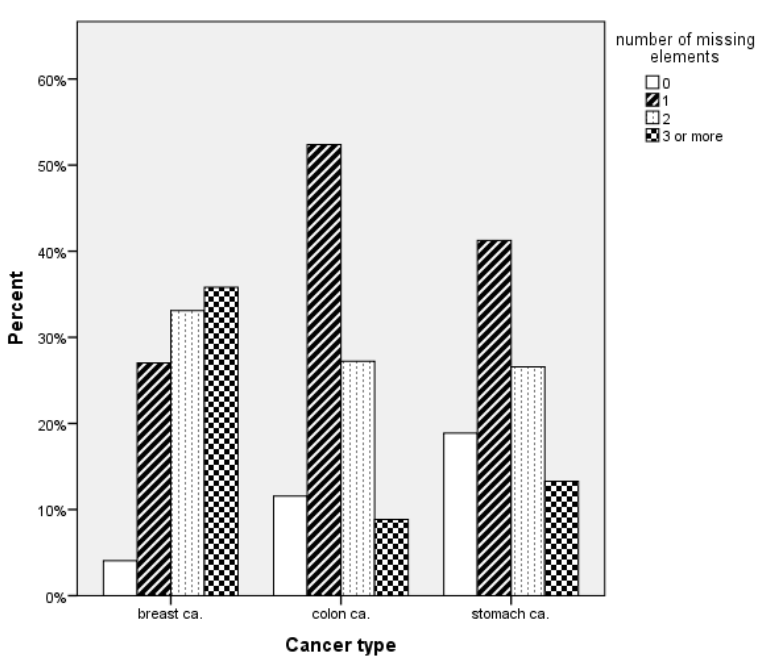

Figure 1. Percentage of Pathology Reports with Missing Elements in Breast, Colon, and Stomach Cancer

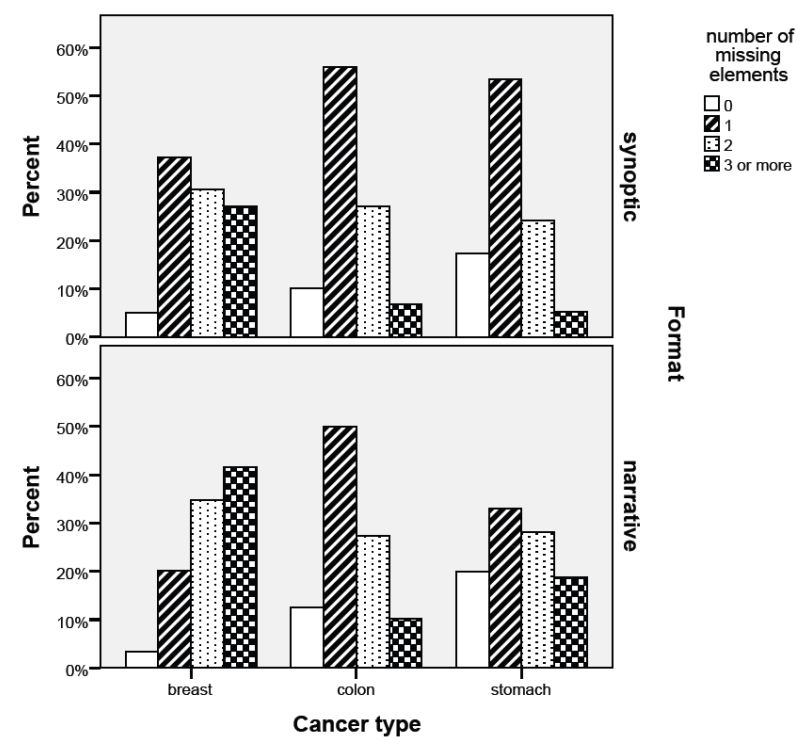

Figure 2. Percentage of Pathology Reports with Missing Elements by Cancer type in Synoptic and Narrative Formats

Table 2. Completeness of pathology reports in synoptic and narrative formats. Data is presented as median (interquartile range).

\begin{tabular}{lccc}
\hline & $\begin{array}{c}\text { Synoptic } \\
\text { format }\end{array}$ & $\begin{array}{c}\text { Narrative } \\
\text { format }\end{array}$ & P value \\
\hline Breast Cancer \% & $82(73-91)$ & $82(73-82)$ & 0.03 \\
Colon Cancer \% & $90(80-90)$ & $90(80-90)$ & 0.76 \\
Stomach Cancer \% & $90(80-90)$ & $90(80-100)$ & 0.7 \\
Overall & $90(80-90)$ & $82(80-90)$ & 0.05 \\
\hline
\end{tabular}

the total cases were complete. Most of them (40.2\%) had one missing element.

Figure 2 shows the percent of reports with missing elements by cancer types in synoptic and narrative formats. For breast cancer, 5.4\% of the synoptic reports were complete. Over $37 \%$ of the synoptic reports had one 
missing element. Only $3.4 \%$ of narrative breast cancer reports were complete. Most of them (41.6\%) lacked 3 or more elements.

For colon cancer, the rates of complete reports were 10.2 and $12.5 \%$ in synoptic and narrative formats, respectively. 56.0 and $50.0 \%$ of the reports lacked one element in synoptic and narrative formats, respectively.

Stomach cancer reports (17.2 and 20.0\%) were complete in synoptic and narrative format, respectively. 53.4 and $33.0 \%$ of the synoptic and narrative stomach cancer reports had one missing element, respectively.

The completeness of reports for colon and stomach cancer was significantly higher than that for breast cancer ( $\mathrm{P}$ value $=0.007,<0.001$, respectively). The difference of completeness between colon and stomach cancer was not significant $(\mathrm{P}$ value $=0.26)$.

Table 2 shows the completeness of reports in synoptic and narrative formats. The completeness of breast cancer reporting in synoptic format was slightly greater than that in narrative format $(\mathrm{P}$ value $=0.03)$. The difference was not significant for colon and stomach cancer reporting. Synoptic format had greater completeness in the total cases $(\mathrm{P}$ value $=0.05)$.

\section{Discussion}

Efforts were made in 1990s to evaluate the adequacy of surgical pathology reports (Kempson, 1992; Zarbo, 1992; Gephardt and Baker, 1996). In an article by Richard Zarbo, 15940 pathology reports of colorectal carcinoma were evaluated for completeness (Zarbo, 1992). He demonstrated that the use of standard report form or checklist was significantly associated with more adequate transmission of information (Zarbo, 1992). In an editorial article commented on this study, Richard Kempson emphasized the needs for the evaluation of surgical pathology reports for completeness (Kempson, 1992). He also suggested the use of checklist reporting style to improve the quality of reports (Kempson, 1992). Both studies (Kempson, 1992; Zarbo, 1992), along with another study (Gephardt and Baker, 1996), demonstrated the insufficiency of pathology reports leading to the introduction of several guidelines (Williams et al., 2015). In 1998, CAP introduced its first edition of protocols for cancer reporting (Williams et al., 2015).

Powsner et al. reported a significant discordance between the intended meaning of pathology reports and the interpretation of these by surgeons. They proposed the use of standardized format to reduce misconception between the pathologist and the clinician (Powsner et al., 2000).

CAP has developed checklists for reporting surgical specimens, available at the CAP website (Goldsmith et al., 2008; Srigley et al., 2009). Similar guidelines were introduced by the American College of Surgeons Commission on Cancer (ACS-CoC) (Goldsmith et al., 2008; Srigley et al., 2009). A surgical pathology report containing all scientifically validated elements mandated by CAP or ACS-CoC was considered complete (Idowu et al., 2010).

This pilot study was conducted to explore the adequacy of pathology reports of breast, colon, and stomach cancer according to CAP checklists. In the current study, many reports lacked the essential elements staging was the most common missing element in the three cancer types and in the total reports, followed by tumor site for breast and stomach cancer, and procedure for colon cancer. The total rate of complete reports was $11.4 \%$. Over $40 \%$ of the reports lacked one mandatory element. This study, similar to the previous reports (Zarbo, 1992), showed a higher rate of completeness for synoptic format reporting.

In an article by Idowu et al. reviewing 2125 cancer reports, 'invasion extent' in breast cancer, and 'vascular invasion' in colorectal cancer were the most common missing elements (Idowu et al., 2010). Over 68\% of their reports were complete. Although, the settings of these studies are not the same; the increased use of checklists in the mentioned study (Idowu et al., 2010) compared with the present study can explain the difference in the rate of complete reports (68.8\% vs. $11.4 \%)$.

In the present study, a variation of completeness of reports for different cancer sites was found. Breast cancer reports had a significant lower rate of completeness. Three or more elements were missing in over $35 \%$ of breast cancer reports.

A complete pathology report also requires the involvement of the clinician. Some mandated elements in reports, that is, the breast quadrant of the lumpectomy specimen cannot be obtained, unless the clinician provides it on the requisition form (Srigley et al., 2009; Nakhleh, 2011). The requisition form must contain information on the tumor location, surgical procedure, and metastasis identified by imaging and laboratory study (Srigley et al., 2009). Data provided on the requisition form is also important to obtain an accurate tumor staging (Srigley et al., 2009). In this study, elements requiring the information on the requisition form (staging, tumor site, and procedure) were commonly missed. This emphasizes the need for standardization of requisition forms and pathology reports.

Another important factor influencing the quality of cancer reporting is the use of a surveillance system before verification of the report (Onerheim et al., 2008; Idowu et al., 2010). Onerheim et al. demonstrated a significant improvement in the quality of breast cancer reporting when a surveillance system was applied (Onerheim et al., 2008). Similarly, in a study by Idowu et al., institutions with a surveillance system for error tracking had a higher report adequacy than that in institutions with no surveillance system (88\% vs. 68\%) (Idowu et al., 2010). None of the hospitals in this study implemented a surveillance system for reporting.

In 2007, CAP introduced the electronic version of the checklists to reduce the limitations of the paperbased ones. Nowadays, many web-based reporting system based on synoptic checklists are available. The Cancer Registry of Norway and the Norwegian Society of Pathology implanted a national electronic checklist for surgical pathology reporting of colorectal carcinoma (Haugland et al., 2011). Casati and Bjugn showed that after implantation of the mentioned electronic checklist, the presence of essential elements in reports was significantly improved (Casati and Bjugn, 2012). Baskovich and Allan introduced a web-based synoptic reporting system based 
Maryam Kadivar and Parnian Kheirkhah Rahimabad

on CAP checklists with simple using and minimized typing necessity and potential errors (Baskovich and Allan, 2011). Pathology reports could benefit from the implication of such systems in hospitals together with a proper monitoring system to ensure the quality of reports.

Although the results of this study cannot be generalized to all institutions; this study supports the need for application of a checklist-style reporting and a surveillance system for early detection of errors. The involvement of clinicians and pathologists to reduce missing elements, such as staging, tumor site, and procedure in the reports was suggested. We hope that this study entice other researchers to further evaluate the adequacy of surgical pathology reports and possible ways to improve it.

\section{References}

Baskovich BW, Allan RW (2011). Web-based synoptic reporting for cancer checklists. J Pathol Inform, 2, 16.

Casati B, Bjugn R (2012). Structured electronic template for histopathology reporting on colorectal carcinoma resections: five-year follow-up shows sustainable long-term quality improvement. Arch Pathol Lab Med, 136, 652-6.

Gephardt GN, Baker PB (1996). Lung carcinoma surgical pathology report adequacy: a College of American Pathologists Q-Probes study of over 8300 cases from 464 institutions. Arch Pathol Lab Med, 120, 922-7.

Goldsmith JD, Siegal GP, Suster S, et al (2008). Reporting guidelines for clinical laboratory reports in surgical pathology. Arch Pathol Lab Med, 132, 1608-16.

Haugland HK, Casati B, Dorum LM, et al (2011). Template reporting matters--a nationwide study on histopathology reporting on colorectal carcinoma resections. Hum Pathol, 42, 36-40.

Idowu MO, Bekeris LG, Raab S, et al (2010). Adequacy of surgical pathology reporting of cancer: a College of American Pathologists Q-Probes study of 86 institutions. Arch Pathol Lab Med, 134, 969-74.

Kempson RL (1992). The time is now. Checklists for surgical pathology reports. Arch Pathol Lab Med, 116, 1107-8.

Messenger DE, McLeod RS, Kirsch R (2011). What impact has the introduction of a synoptic report for rectal cancer had on reporting outcomes for specialist gastrointestinal and nongastrointestinal pathologists? Arch Pathol Lab Med, 135, 1471-5.

Nakhleh RE (2006). What is quality in surgical pathology? $J$ Clin Pathol, 59, 669-72.

Nakhleh RE (2011). Quality in surgical pathology communication and reporting. Arch Pathol Lab Med, 135, 1394-7.

Onerheim R, Racette P, Jacques A, et al (2008). Improving the quality of surgical pathology reports for breast cancer: a centralized audit with feedback. Arch Pathol Lab Med, 132, 1428-31.

Powsner SM, Costa J, Homer RJ (2000). Clinicians are from Mars and pathologists are from Venus. Arch Pathol Lab Med, 124, 1040-6.

Srigley JR, McGowan T, Maclean A, et al (2009). Standardized synoptic cancer pathology reporting: a population-based approach. J Surg Oncol, 99, 517-24.

Williams CL, Bjugn R, Hassell LA (2015). Current status of discrete data capture in synoptic surgical pathology and cancer reporting. Pathol Laboratory Med Intl, 7, 11- 22.

Zarbo RJ (1992). Interinstitutional assessment of colorectal carcinoma surgical pathology report adequacy. A College of American Pathologists Q-Probes study of practice patterns from 532 laboratories and 15,940 reports. Arch Pathol Lab Med, 116, 1113-9. 